\title{
Clinical-Cytological-Grading and phenotyping in patients with chronic rhinosinusitis with nasal polyps: the relevance in clinical practice
}

\author{
Matteo Gelardi ${ }^{1}$, Giuseppe Porro ${ }^{2}$, Vitaliano Quaranta $^{3}$, Nicola Quaranta ${ }^{2}$, Michele Cassano ${ }^{1}$, \\ Giorgio Ciprandi ${ }^{4}$, Italian Study Group on CRSwNP* \\ ${ }^{1}$ Department of Otolaryngology, University of Foggia; ${ }^{2}$ Otolaryngology, Department of Basic Medical Science, \\ Neuroscience and Sensory Organs, University of Bari; ${ }^{3}$ Pulmonology Unit, Ospedale Di Venere, Bari; ${ }^{4}$ Allergist consultant, \\ Casa di Cura Villa Montallegro, Genoa, Italy
}

\begin{abstract}
Chronic rhinosinusitis (CRS) includes two main phenotypes: without nasal polyps (CRSsNP) and with nasal polyps (CRSwNP). CRSwNP may be associated with comorbidity, mainly concerning asthma, aspirin intolerance, and allergy. CRSwNP patients may also be evaluated by clinical-cytological grading (CCG). The current study investigated the prevalence and characteristics of the dif-
\end{abstract}

Correspondence: Giorgio Ciprandi, Via Boselli 5, 16146 Genoa, Italy. E-mail: gio.cip@libero.it

Key words: chronic rhinosinusitis with nasal polyps, asthma, clinicalcytological-grading, cytological phenotype, comorbidity, real-world.

Authors' Contributions: All the authors made a substantive intellectual contribution. All the authors have read and approved the final version of the manuscript and agreed to be accountable for all aspects of the work.

Conflict of interest: The authors declare that they have no competing interests, and all authors confirm accuracy.

Funding: There was no sponsorship.

Availability of data and materials: The datasets used and/or analyzed during the current study are available from the corresponding author on reasonable request.

Ethics approval: The Review Board of each clinical center approved the procedures used in this study.

Informed consent: Written informed consent was obtained from the patients.

Received for publication: 19 March 2020.

Accepted for publication: 4 May 2020.

${ }^{\circ}$ Copyright: the Author(s), 2020

Licensee PAGEPress, Italy

Monaldi Archives for Chest Disease 2020; 90:1277

doi: 10.4081/monaldi.2020.1277

This article is distributed under the terms of the Creative Commons Attribution Noncommercial License (by-nc 4.0) which permits any noncommercial use, distribution, and reproduction in any medium, provided the original author(s) and source are credited. ferent CCG and phenotypes in CRSwNP outpatients examined in clinical practice. This retrospective cross-sectional study enrolled 791 consecutive CRSwNP outpatients (424 males, mean age 48.8 years). In the total population, asthma was a common comorbidity $(30.8 \%)$ as well as aspirin intolerance $(24.8 \%)$, and allergy (50.8\%). As concerns CCG-grading, 210 (26.5\%) outpatients had low-grade, 366 (46.3\%) medium, and 215 (27.2\%) high. As regards cytological phenotypes, $87(11 \%)$ had neutrophilic type, 371 (46.3\%) eosinophilic, 112 (14.2\%) mast cell, and 221 (27.9\%) mixed. High-grade CCG was significantly associated with more frequent asthma, aspirin intolerance, allergy, recurrent surgery, and mixed cytological phenotype. Low-grade CCG was characterized by fewer comorbidities and operations, and neutrophilic phenotype. Therefore, the present study confirmed that CCG is a useful tool in the management of outpatients with CRSwNP. CRSwNP is frequently associated with asthma, aspirin intolerance, and allergy comorbidity. High-grade CCG is frequently characterized by a mixed cytological phenotype, thus, by more severe progress. These real-world outcomes underline that CRSwNP deserves adequate attention for careful management and optimal identification of the best-tailored therapy; CCG and cytological phenotyping could be fruitful tools in clinical practice. Asthma and aspirin intolerance should be adequately investigated in all CRS patients.

\section{Introduction}

Chronic rhinosinusitis (CRS) affects about $10-12 \%$ of the European population [1]. Based on the endoscopy and computed tomography (CT) findings, CRS may be classified into two main phenotypes: CRS with nasal polyposis (CRSwNP) and CRS without nasal polyposis (CRSsNP) [2]. CRSwNP is defined by the presence of nasal polyps and by signs and symptoms lasting longer than 8-12 weeks [2,3]. Nasal polyps are benign oedematous masses in the nasal cavities, paranasal cavities, or both. Their

\footnotetext{
*Italian Study Group on CRSwNP:

Luigi Alonzi, Arturo Arnone Caruso, Francesco Barbara, Michele Barbara, Paolo Castelnuovo, Giovanna Ciancio, Andrea Ciofalo, Michele Curatolo, Nicola De Candia, Carlo De Luca, Valentina De Robertis, Valentina Di Benedetto, Valeria Fiore, Lucia Iannuzzi, Clara Imperatore, Cristiano Lingua, Giuseppe Lombardo, Alberto Macchi, Michela Mancini, Alessandro Maselli del Giudice, Patrizia Mola, Mario Notargiacomo, Antonio Palma, Piero Pecoraro, Giuseppe Pellitteri, Krizia Piccininni, Massimo Rinaldi, Valentina Rossi.
} 
occurrence depends on an exaggerated inflammatory reaction. As nasal polyps occupy space into nasal cavities, they can cause nasal obstruction, rhinorrhoea, postnasal drip, and hypo/anosmia [4]. The CRSwNP overall prevalence has been approximately estimated to be $2 \%$ to $4 \%$ of the general population. It has been calculated that approximately $30 \%$ of patients suffering from CRSwNP also have asthma [1-3]. Patients with both diseases, i.e., the CRSwNPasthma phenotype, have a weak therapeutic response and a high recurrence rate, so the disease is more severe and challenging to treat [5]. CRSwNP-asthma phenotype also induces a severe impairment of quality of life (QoL) and causes a substantial financial burden for society [3]. Moreover, CRSwNP has frequently associated also with aspirin intolerance [6]. In particular, the coexistence of CRSwNP, asthma, and aspirin intolerance (the so-called aspirin triad or Widal's Syndrome or Samter's Syndrome) is a relevant adverse prognostic factor with a higher risk of postsurgical recurrences [6]. In this regard, CRSwNP may be, in turn, classified considering different phenotypes, including comorbidity, endoscopic outcomes, X-ray features, and cytological pattern [7]. In particular, a clinical-cytological grading (CCG) has been proposed for defining the best management strategy, to individuate a peculiar prognostic index of relapse, a personalized medical approach, and the association with olfactory dysfunction $[8,9]$. In this regard, recent technological advances, mainly in the fields of genetics and engineering, expanded the information concerning the phenotypes and the endotypes of chronic respiratory disorders, generally including the type of inflammation and immune response [10]. This information could enable more targeted, effective, and efficient Precision Medicine (PM). PM refers to the "ability to classify individuals into subpopulations that differ in their susceptibility to a particular disease, in the biology or prognosis of those diseases they may develop, or in their response to a specific treatment" [11]. Consequently, PM allows us to stratify patients into subgroups and to tailor treatment based on their peculiar pheno/endotypes [12]. This approach has been defined with the term Personalized Medicine [13]. Based on this background, we aimed to investigate a large group of Italian outpatients with CRSwNP, who were recruited in a real-world setting. The purpose was to define the prevalence and the characteristics of the different CCG and phenotypes in CRSwNP outpatients examined in clinical practice.

\section{Materials and Methods}

\section{Study population}

A consecutive series of outpatients was visited at 26 Italian Rhinology Centers; after the initial screening, the outpatients were enrolled in this cross-sectional study conducted from June 2018 to June 2019

The inclusion criteria were: i) age $\geq 18$ yrs old, ii) documented diagnosis of CRSwNP, and iii) informed written consent.

The exclusion criteria were: i) severe anatomic defect of the nasal cavity and/or the nasal pyramid, and ii) concomitant diseases or treatments (including systemic corticosteroids) that could interfere with the interpretation of the results.

The Review Board of each clinical center approved the procedures used in this study.

\section{Study design}

All patients were evaluated by clinical history, objective examination, fiber-optic endoscopy, nasal cytology, and skin prick test.
The diagnosis of CRSwNP was performed according to validated criteria stated by European and International guidelines [3].

\section{Outcome}

The primary outcome of the current study was to stratify a large group of Italian outpatients with CRSwNP according to the 3 degrees of CCG, such as low ( $\leq 3)$, medium (4-6), and high $(\geq 7)$, and the cytological phenotypes [13].

\section{Variables}

Clinical characteristics included age, gender, family predisposition to nasal polyps, asthma, and allergy. Age at onset was also evaluated as well as the number of surgery operations for NP. CRSwNP comorbidity included allergy, asthma, aspirin intolerance, and the aspirin triad, such as CRSwNP associated with asthma and aspirin intolerance. Satisfaction for surgery and medical treatment was assessed by the visual analog scale (VAS), where 0 means the lowest level of satisfaction and ten the maximal level.

Nasal endoscopy was carried out by a $3.4 \mathrm{~mm}$ diameter flexible fiberscope (Vision-Sciences ${ }^{\circledR}$ ENT-2000). Nasal polyp endoscopic 4-grade classification proposed by Meltzer was adopted [14].

Nasal cytology includes sampling, processing, and microscope reading. Sampling requires the collection of cells from the surface of the middle portion of the inferior turbinate by a sterile disposable curette. The procedure is performed under anterior rhinoscopy, with an appropriate light source, and it is painless. The sample obtained is immediately smeared on a glass slide, air-dried, and stained with May-Grünwald-Giemsa (MGG) for $30 \mathrm{~min}$. The stained sample is read at optical microscopy, with a 1000x objective with oil immersion. Fifty fields are considered the minimum number to identify a sufficient number of cells. The count of each cell type was expressed by a semi-quantitative grading, as previously described [8]. The predominant type of inflammatory cell, present in the sample, was considered, such as neutrophilic, eosinophilic, mast cell infiltrates, and mixed cellularity (eosinophil and mast cell). In this way, four cytologic phenotypes were identified: neutrophilic, eosinophilic, mast cell, and mixed, according to validated methodology [14].

Skin prick test was performed, as stated by the European Academy of Allergy and Clinical Immunology [15]. The allergen panel consisted of the following: house-dust mites (Dermatophagoides farinae and Dermatophagoides pteronyssinus), cats, dogs, grasses mix, Compositae mix, P. Judaica, birch, hazel trees, olive trees, cypress, Alternaria tenuis, Cladosporium, and Aspergilli mix. The concentration of allergen extracts was 100 immune reactivity/mL (Stallergenes-Greer Italia, Milan, Italy). A histamine solution in distilled water $(10 \mathrm{mg} / \mathrm{mL})$ was used as the positive control, and the glycerol-buffer diluent of the allergen preparations was used as the negative control. Each patient was skin tested on the volar surface of the forearm using 1-mm prick lancets. The skin reaction was recorded after 15 minutes by evaluating the skin response in comparison with the wheal given by the positive and the negative control. Wheel diameter of at least $3 \mathrm{~mm}$ was considered a positive reaction. Allergy was defined by the consistency between sensitization and symptom occurrence after the exposure to the sensitizing allergen [16].

Clinical-Cytological Grading has been previously described in detail elsewhere [8,9]. Briefly, CCG is a score based on both nasal cytology findings and some co-morbidities, including asthma, allergy, and aspirin intolerance. To each variable a score value was assigned: neutrophilic infiltrate was scored as 1, mast cell infiltrate was scored 1, eosinophilic infiltrate was scored 2, eosinophilic + 
mast cell was scored 4; similarly, aspirin intolerance was scored 1, asthma 2, allergy 2, aspirin intolerance + asthma 3 . The CCG was composed as the sum of these individual scores. A global score between $1-3$ is considered low grade, $4-6$ is medium, and $\geq 7$ is high $[8,9]$.

\section{Statistical analysis}

Continuous variables were given as means with standard deviations or medians and interquartile ranges (IQR), and categorical variables as the number of subjects and percentage values. The three classes of CCG (low, medium, and high) were considered as the primary outcome. Any statistically significant difference in the mean values among patients with different CCG scores was evaluated by ANOVA followed by Bonferroni post-hoc test.

Statistical significance was set at $p<0.05$, and the analyses were performed using GraphPad Prism software, GraphPad Software Inc, CA, USA and Epi-Info statistical software (Centers for Disease Control and Prevention, Atlanta, GA, USA) were used.

\section{Results}

This study included 791 outpatients, mean age $45.88 \pm 16.26$ years, 424 males, and 367 females. Demographic and clinical characteristics are reported in Table 1 . There was a slight preponderance of males: $53.6 \%$ versus $46.4 \%$. Family predisposition to nasal polyps was present in $205(25.9 \%)$ outpatients, to asthma in 242 $(30.6 \%)$, and allergy in $305(38.6 \%)$. The mean age at onset was

Table 1. Demographic and clinical characteristics of the outpatients with CRSwNP.

\begin{tabular}{lc}
\hline Total outpatients (n=791) & \\
Age (years) Mean \pm SD & $45.88 \pm 16.26$ \\
Males N (\%) & $424(53.6)$ \\
\hline Females & $367(46.4)$ \\
Family predisposition to nasal polyps N (\%) & $205(25.9)$ \\
\hline Family predisposition to asthma N (\%) & $242(30.6)$ \\
Family predisposition to allergy N (\%) & $305(38.6)$ \\
\hline Age at onset (years) Mean \pm SD & $39.86 \pm 14.42$ \\
Asthma comorbidity N (\%) & $244(30.8)$ \\
\hline Aspirin intolerance N (\%) & $196(24.8)$ \\
Aspirin triad N (\%) & $165(20.9)$ \\
\hline Allergy N (\%) & $402(50,8)$ \\
Operations median (IQR) & $1(0-1)$ \\
\hline Satisfaction for surgery (VAS) Median (IQR) & $7(5-8)$ \\
Satisfaction for medical treatment (VAS) Median (IQR) & $7(6-8)$ \\
\hline CCG N (\%) & \\
Low & $210(26.5)$ \\
Medium & $366(46.3)$ \\
High & $215(27.2)$ \\
Cytological phenotypes N (\%) & \\
Neutrophilic & $87(11.0)$ \\
Eosinophilic & $371(46.9)$ \\
Mast cell & $112(14.2)$ \\
Mixed (eosinophils + mast cells) & $221(27.9)$ \\
\hline
\end{tabular}

$39.86 \pm 14.42$ years. Therefore, the mean duration of the disease was about six years. Asthma comorbidity was found in 244 (30.8\%) outpatients, aspirin intolerance in $196(24.8 \%)$, the aspirin triad in $165(20.9 \%)$, and allergy in 402 (50.8\%). Findings concerning operations, and satisfaction grades are reported in Table 1.

The CCG grade was low in $210(26.5 \%)$ outpatients, medium in $366(46.3 \%)$, and high in 215 (27.2\%). Neutrophilic phenotype was identified in $87(11 \%)$ outpatients, eosinophilic phenotype in 371 (46.9\%), mast cell phenotype in $112(14.2 \%)$, and mixed (eosinophils and mast cells) phenotype in 221 (27.9\%).

The comparison among CCG grades is reported in Table 2. The age was significantly higher in outpatients with the low CCG grade. There was a preponderance of the male gender in the low CCG grade. Outpatients with high CCG grade had greater family predisposition to allergy, lower age at onset, more prevalent asthma comorbidity, more frequent aspirin intolerance, allergy, and aspirin triad.

The number of operations was significantly different among CCG grades. There was a significant $(p=0.002)$ difference in the level of satisfaction for medical treatments: more evident between the medium and high-grade CCG.There was a significant difference in the cytological phenotypes among CCG grades (Figure 1). A most common neutrophilic phenotype characterized the lowgrade CCG in comparison with the medium-grade and the highgrade; no subject had the mixed phenotype in this group. The medium-grade CCG had the highest prevalence of eosinophilic phenotype in comparison with the low-grade and the high-grade. The high-grade CCG had the highest prevalence of the mixed phenotype in comparison with the low grade and the medium-grade; no outpatient had the neutrophilic phenotype, and only six subjects had the mast cell phenotype.

\section{Discussion}

The current study, conducted in real-world settings and scattered across Italy, provided some outcomes that could have clinical relevance in daily practice.

Asthma comorbidity was rather common as about $30 \%$ of outpatients were asthmatic as well as about half of them were allergic. These findings are consistent with the concept of united airway

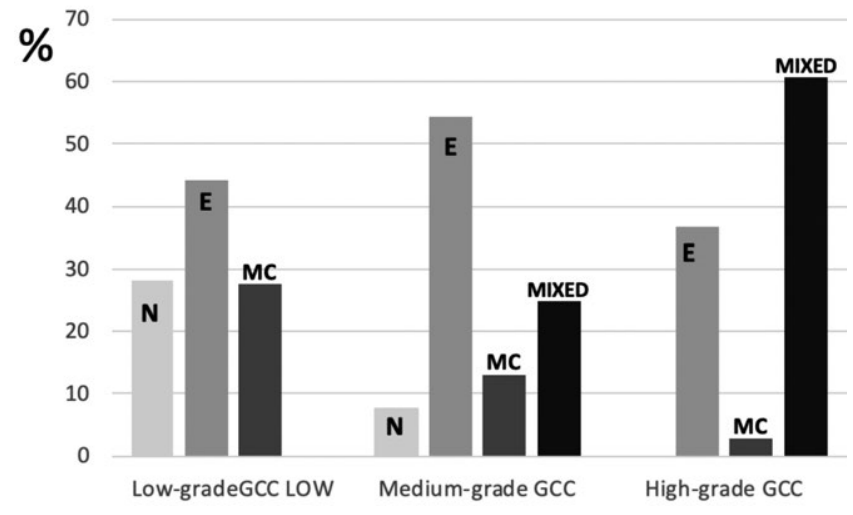

Figure 1. Frequency distribution among CCG grades considering the different cytological phenotypes: $N$, neutrophilic; $E$, eosinophilic; MC, mast cell; Mixed,eosinophilic+mast cell. 
diseases, such as rhinitis, rhinosinusitis, and asthma, which are frequently coexisting $[17,18]$. In particular, the CRSwNP-asthma phenotype, mainly if associated with aspirin intolerance, is particularly severe and needs careful management [7].

The distribution of the outpatients according to the CCG grading showed that the most frequent grade was the medium (about half), whereas the other grades were similar (about $1 / 4$ each one). The nasal cytology assessment allowed us to identify four cytological phenotypes: eosinophilic, the most common as affected about the half of the sample, mixed (27.9\%), mast cell (14.2\%), and neutrophilic (11\%). Knowing these characteristics is very important in the light of Precision Medicine. Pheno/endotypingdriven is crucial in clinical practice to tailor the best treatment for every patient [19]. However, the bulk of the evidence on this topic derives from studies or trials, recruiting selected patients whose results have limited applicability as do not reflect what is commonly observed in clinical practice [20]. Indeed, high-level evidence deriving from very selected subjects could not concern all patients. There is consequently the need to recruit a CRSwNP population in a real-world setting.

The primary outcome showed that CCG was able to detect significant differences among the grades that could be translated into clinical practice. Outpatients with high-grade CCG had more frequent asthma and aspirin intolerance comorbidity as well as associated allergy. These associations were also consistent with the family predispositions to have asthma and/or allergy. These findings underline the relevance of type 2 inflammation in the pathogenesis of upper and lower airways diseases [21-23]. Moreover, outpatients with high-grade CCG had more operations and consequently greater dissatisfaction with surgery. More interestingly, the cytological phenotyping underlined the relevance of CCG. Indeed, high-grade CCG very frequently was associated with a mixed phenotype. Usually, patients with mixed nasal infiltrate present severe airway disorder characterized by more intense symptoms and reduced responsiveness to standard treatments [1]. On the other hand, no high-grade CCG outpatient had the neutrophilic phenotype, as well as no low-grade CCG outpatient, had mixed phenotype. This finding suggests a severity gradient of CRSwNP from the lower grade to the higher one. Consistently high-grade CCG was associated with a series of adverse prognostic factors that could predict an unfavorable outcome, characterized by refractory to both medical and surgery standard options. In this regard, the potential use of biologics could pave the way to new therapeutic strategy [24-28]. Therefore, a thorough workup is mandatory to individuate the better treatment. An adequate pheno/endotyping could identify individual therapy.

The present study has some limitations, including the crosssectional design, the lack of mediators' assessment, and the absence of imaging documentation. On the other hand, the current study was performed in a real-world setting. The real-world studies as they may provide information more adherent to the daily practice that randomized controlled trial that involves selected patient populations that rarely mirror the real situation $[29,30]$. Finally, the sample was large and well-distributed across Italy.

\section{Conclusions}

The present study confirmed that CCG is a useful tool in the management of outpatients with CRSwNP. CRSwNP is frequently associated with asthma, aspirin intolerance, and allergy comorbidity. High-grade CCG is frequently characterized by a mixed cytological phenotype, thus, by severe progress. These outcomes underline that CRSwNP deserves adequate attention to careful management and optimal identification of the best-tailored thera-

Table 2. Stratification of the outpatients with CRSwNP based on the CCG grade.

\begin{tabular}{|c|c|c|c|c|}
\hline Variables & $\begin{array}{c}\text { CCG low } \\
\mathrm{N}=210(26.5 \%)\end{array}$ & $\begin{array}{l}\text { CCG medium } \\
\mathrm{N}=366(46.3 \%)\end{array}$ & $\begin{array}{c}\text { CCG high } \\
\mathrm{N}=214(27.2 \%)\end{array}$ & $\mathbf{p}$ \\
\hline Age (years), mean \pm SD & $48.46 \pm 16.89^{* \circ}$ & $43.96 \pm 16.30$ & $46.60 \pm 15.20$ & 0.004 \\
\hline Males, N (\%) & $128(61.0)^{* * 0}$ & $178(48.6)$ & $118(54.9)$ & 0.016 \\
\hline Family predisposition to NP, N (\%) & $43(20.5)$ & $102(27.9)$ & $60(27.9)$ & 0.110 \\
\hline Family predisposition to asthma, N (\%) & $54(15.7)$ & $116(31.7)$ & $72(33.5)$ & 0.182 \\
\hline Family predisposition to allergy, N (\%) & $59(28.1)^{* *} \S * * * \circ$ & $143(39.1)^{* \wedge}$ & $103(47.9)$ & 0.000 \\
\hline Age at onset (years) mean (SD) & $42.59 \pm 15.28^{* \circ * *} \S$ & $39.01 \pm 14.60$ & $38.63 \pm 12.88$ & 0.006 \\
\hline Asthma comorbidity, $\mathrm{N}(\%)$ mean (SD) & $29(13.8)^{* * *} \S * * \circ$ & $86(23.5)^{* * \wedge}$ & $129(60.0)$ & 0.000 \\
\hline Aspirin intolerance, $\mathrm{N}(\%)$ mean (SD) & $38(18.1)^{* * * * * *} \S$ & $82(22.4)$ & $76(35.3)$ & 0.000 \\
\hline Aspirin triad, N (\%) mean (SD) & $25(11.9)^{* * \circ * * \S}$ & $75(20.5)$ & $65(30.2)$ & 0.000 \\
\hline Allergy, N (\%) mean (SD) & $2(1)^{* * * \circ * * * \S}$ & $212(57.9)^{* * *}$ & $188(87.4)$ & 0.000 \\
\hline Operations median (IQR) & $1[0-1]^{* 0 * * \S}$ & $1[0-1]$ & $1[1-1]$ & 0.000 \\
\hline Satisfaction for surgery, median (IQR) & $7[7-9]$ & $6[7-9]$ & $7[5-9]$ & 0.388 \\
\hline Satisfaction for medical treatment, median (IQR) & $7[6-7]^{* \circ}$ & $7[6-8.25]$ & $7[5-8]$ & 0.002 \\
\hline $\begin{array}{l}\text { Cytological phenotypes N (\%) mean (SD) } \\
\text { neutrophilic } \\
\text { eosinophilic } \\
\text { mast cell } \\
\text { mixed }\end{array}$ & $\begin{array}{c}59(28.1)^{* * \circ * * * \S} \\
93(44.3)^{* * \circ *} \S \\
58(27.6)^{* * *} \S \\
0(0)^{* * * * * * \S}\end{array}$ & $\begin{array}{l}28(7.7)^{* * \wedge} \\
199(54.4)^{* \wedge} \\
48(13.1)^{* * \wedge} \\
91(24.9)^{* \wedge}\end{array}$ & $\begin{array}{c}0(0) \\
79(36.7) \\
6(2.8) \\
130(60.6)\end{array}$ & 0.000 \\
\hline
\end{tabular}

$\mathrm{NP}$, nasal polyps. At post-hoc analysis: ${ }^{\mathrm{C} C G}$ low us medium; ${ }^{\circledR} \mathrm{CCG}$ low us high; ${ }^{\wedge} \mathrm{CCG}$ medium vs high; ${ }^{*} \mathrm{p}<0.05 ;{ }^{* *} \mathrm{p}<0.001 ;{ }^{* * *} \mathrm{p}<0.0001$. 
py, and, in this regard, CCG could be a fruitful tool. In particular, considering the estimated prevalence of CRSwNP and the current findings, it could be reasonably expected that about 600.000 Italians have CRSwNP with high-grade CCG. Therefore, intercepting the most severe patients and launching an early and tailored treatment, including the new biologics, could positively modify the natural history and improve the quality of life. In this regard, asthma and aspirin intolerance should be adequately investigated in all CRS patients.

\section{References}

1. Hastan D, Fokkens WJ, Bachert C, et al. Chronic rhinosinusitis in Europe - an underestimated disease. A GA(2)LEN study. Allergy 2011;66:1216-23.

2. Fokkens WJ, Lund VJ, Mullol J, et al. EPOS 2012: European position paper on rhinosinusitis and nasal polyps 2012. A summary for otorhinolaryngologists. Rhinology 2012;50:1-12.

3. Orlandi RR, Kingdom TT, Hwang PH, et al. International Consensus Statement on Allergy and Rhinology: Rhinosinusitis. Int Forum Allergy Rhinol 2016;6:S22-209.

4. Lund VJ, Mackay IS. Staging in rhinosinusitis. Rhinology 1993;31:183-4.

5. Lin DC, Chandra RK, Tan BK, et al. Association between severity of asthma and degree of chronic rhinosinusitis. Am J Rhinol Allergy 2011;25:205-8.

6. Podwysocka M, Dąbrowska K, Fendler W, et al. Analysis of the impact of bronchial asthma and hypersensitivity to aspirin on the clinical course of chronic sinusitis with nasal polyps. Otolaryngol Pol 2019;73:37-43.

7. Meltzer EO, Hamilos DL, Hadley JA, et al. Rhinosinusitis: developing guidance for clinical trials. Rhinosinusitis Initiative. J Allergy Clin Immunol 2006;118:S17-61.

8. Gelardi M, Iannuzzi L, De Giosa M, et al. Non-surgical management of chronic rhinosinusitis with 1 nasal polyps based on clinical cytological grading: a precision medicine-based approach. Acta ORL Ital 2017;37:38-45.

9. Gelardi M, Piccininni K, Quaranta N, et al. Olfactory dysfunction in patients with chronic rhinosinusitis with nasal polyps is associated with Clinical-Cytological Grading severity. Acta Otorhinolaryngol Ital 2019;39:329-35.

10. Patou J, Holtappels G, Affleck K, et al. Enhanced release of IgE-dependent early phase mediators from nasal polyp tissue. J Inflamm (Lond) 2009;6:11.

11. Timmerman L. What is in a name? A lot, when it comes to 'precision medicine'. Xconomy Internet]. 4 February 2013. Available from: https://xconomy.com/national/2013/02/04/ whats-in-a-name-a-lot-when-it-comes-to-precision-medicine/

12. Kaur R, Chupp G. Phenotypes and endotypes of adult asthma: moving toward precision medicine. J Allergy Clin Immunol 2019;144:1-12.

13. Guilleminault L, Ouksel H, Belleguic C, et al. Personalised medicine in asthma: from curative to preventive medicine. Eur Respir Rev 2017;26:143.

14. Ciprandi G, Silvestri M. Standardization of the nasal cytology in the work-up of allergic rhinitis. Ann All Asthma Immunol 2019;123:213-6.

15. Bousquet J, Heinzerling L, Bachert C, et al. Global Allergy and Asthma European Network, author. A practical guide to skin prick tests in allergy to aeroallergens. Allergy 2012;67:18-24.

16. Ciprandi G, Marseglia GL, Klersy C, et al. Relationships between allergic inflammation and nasal airflow in children with persistent allergic rhinitis due to mite sensitization. Allergy 2005;60:957-60.

17. Passalacqua G, Ciprandi G. Pasquali M, et al. An update on the asthma-rhinitis link. Curr Opin Allergy Clin Immunol 2004;4: 177-83.

18. Ciprandi G, Caimmi D, Miraglia del Giudice M, et al. Recent developments in united airways disease. Allergy Asthma Immunol Res 2012;4:171-7.

19. Tay TR, Hew M. Comorbid "treatable traits" in difficult asthma. Current evidence and clinical evaluation. Allergy 2018;73:1369-82.

20. Oyinlola JO, Campbell J, Kousoulis AA. Is real-world evidence influencing practice? A systematic review of CPRD research in NICE guidance. BMC Health Serv Res 2016;16:299.

21. Southworth T, Pattwell C, Khan N, et al. Increased type 2 inflammation post rhinovirus infection in patients with moderate asthma. Cytokine 2019;125:154857.

22. Kalinauskaite-Zukauske V, Januskevicius A, Janulaityte I, et al. Expression of eosinophil $\beta$ chain-signaling cytokines receptors, outer-membrane integrins, and type 2 inflammation biomarkers in severe non-allergic eosinophilic asthma. BMC Pulm Med 2019;19:158.

23. Edris A, De Feyter S, Maes T, et al. Monoclonal antibodies in type 2 asthma: a systematic review and network meta-analysis. Respir Res 2019;20:179.

24. Avdeeva K, Fokkens W. Precision medicine in chronic rhinosinusitis with nasal polyps. Curr Allergy Asthma Rep 2018; $18: 25$.

25. Ren L, Zhang N, Zhang L, et al. Biologics for the treatment of chronic rhinosinusitis with nasal polyps - state of the art. World Allergy Organ J 2019;12-100050.

26. Vlastos I, Gkouskou K, Doulaptsi M, et al. Precision medicine in rhinosinusitis. Curr Allergy Asthma Rep 2019;19:12.

27. Bachert C, Han JK, Desrosiers M, et al. Efficacy and safety of dupilumab in patients with severe chronic rhinosinusitis with nasal polyps (LIBERTY NP SINUS-24 and LIBERTY NP SINUS-52): results from two multicentre, randomized, doubleblind, placebo-controlled, parallel-group phase 3 trials. Lancet 2019;394:1638-50.

28. Stevens WW. A new treatment for chronic rhinosinusitis with nasal polyps. Lancet 2019;394:1595-7.

29. US Food and Drug Administration. Use of real-world evidence to support regulatory decision-making for medical devices. Guidance for industry and Food and Drug Administration staff. August 31, 2017. Available from: https://www.fda.gov/regulatory-information/search-fda-guidance-documents/use-real-worldevidence-support-regulatory-decision-making-medical-devices

30. Sherman RE, Anderson SA, Dal Pan GJ, et al. Real-world evidence - what is it and what can it tell us? N Engl J Med 2016; 375:2293-7. 